\title{
ON THE EXISTENCE OF MULTIPLE POSITIVE SOLUTIONS FOR A CERTAIN CLASS OF ELLIPTIC PROBLEMS
}

\author{
ALEKSANDRA ORPEL \\ Faculty of Mathematics, University of Łódż, Banacha 22, 90-238 Eódż, Poland \\ E-mail: orpela@math.uni.lodz.pl
}

\begin{abstract}
We investigate the existence of solutions for the Dirichlet problem including the generalized balance of a membrane equation. We present a duality theory and variational principle for this problem. As one of the consequences of the duality we obtain some numerical results which give a measure of a duality gap between the primal and dual functional for approximate solutions.
\end{abstract}

1. Introduction. Let us consider functions $G$ and $H$ satisfying the following assumptions:

Q: $q$ is even and $q \geq 2$;

$\Omega: \Omega$ is a bounded domain of $R^{n}$ having a piecewise locally Lipschitz boundary;

H1: $H: \Omega \times R^{n} \rightarrow R$ is a Carathéodory function, $H(y, \cdot)$ is convex and Gateaux differentiable for a.e. $y \in \Omega$;

H2: there exist constants $b_{1}, b_{2}>0$ and functions $k_{1}, k_{2} \in L^{q}(\Omega, R)$ such that for a.e. $y \in \Omega$ and all $z \in R^{n}$

$$
\frac{b_{1}}{q}|z|^{q}+k_{1}(y) \leq H(y, z) \leq \frac{b_{2}}{q}|z|^{q}+k_{2}(y)
$$

H3: $\operatorname{div}\left(H_{z}(y, 0)\right)=0$ for a.e. $y \in \Omega$;

G1: there exist $z_{1}, z_{0} \in W_{0}^{1, q}(\Omega, R) \cap L^{\infty}(\Omega, R)$ such that $0<z_{0}(y)<z_{1}(y)$ for a.e. $y \in \Omega, \operatorname{div}\left(H_{z}\left(\cdot, \nabla z_{0}(\cdot)\right) \in L^{\infty}(\Omega, R)\right.$ and

$$
-G_{x}\left(y, z_{1}(y)\right) \geq \operatorname{div}\left(H_{z}\left(y, \nabla z_{0}(y)\right) \text { a.e. in } \Omega\right. \text {; }
$$

G2: $G(y, \cdot)$ is convex and belongs to $C^{1}(\tilde{I}, R)$ for a.e. $y \in \Omega, G(\cdot, x)$ is measurable on $\Omega$ for all $x \in \tilde{I}$ with $\tilde{I}$ being a certain closed neighborhood of the interval $I=\left[0, \operatorname{ess}_{\sup } \operatorname{su}_{1} z_{1}(y)\right]$ for a.e. $y \in \Omega$;

2000 Mathematics Subject Classification: 35J20, 35J25.

Key words and phrases: nonconvex elliptic Dirichlet problems, positive solutions, duality method, variational method.

The paper is in final form and no version of it will be published elsewhere. 
G3: $G_{x}$ is nonnegative in $\tilde{I}$ for a.e. $y \in \Omega$;

G4: $\int_{\Omega} G_{x}(y, 0) d y \neq 0, \int_{\Omega} G(y, 0) d y>0$ and $\int_{\Omega} G\left(y, \operatorname{ess~sup}_{y \in \Omega} z_{1}(y)\right) d y<\infty$.

We shall investigate the following class of elliptic problems which generalize the balance of a membrane equation:

$$
\left\{\begin{array}{l}
-\operatorname{div}\left(H_{z}(y, \nabla x(y))\right)=G_{x}(y, x(y)) \quad \text { for a.e. } y \in \Omega, \\
x \in W_{0}^{1, q}(\Omega, R)
\end{array}\right.
$$

where $H_{z}(y, z)=\left[\frac{d}{d z_{1}} H(y, z), \ldots, \frac{d}{d z_{n}} H(y, z)\right]$ for $z=\left[z_{1}, \ldots, z_{n}\right] \in R^{n}$ and $G_{x}(y, x)=$ $\frac{d}{d x} G(y, x)$ for $x \in R, y \in \Omega, q \geq 2$. By a (weak) solution of this problem we understand an element $x \in W_{0}^{1, q}(\Omega, R)$ such that for all $\varphi \in C_{0}^{\infty}(\Omega, R)$

$$
\left\{\begin{array}{l}
\int_{\Omega} H_{z}(y, \nabla x(y)) \nabla \varphi(y) d y=\int_{\Omega} G_{x}(y, x(y)) \varphi(y) d y \quad \text { for a.e. } y \in \Omega, \\
x \in W_{0}^{1, q}(\Omega, R) \text { and } \operatorname{div}\left(H_{z}(\cdot, \nabla x(\cdot))\right) \in L^{q^{\prime}}(\Omega, R) .
\end{array}\right.
$$

The aim of this paper is to show that under the above assumptions, (2) possesses solutions from a known pre-specified interval of the positive axis. The question of existence of solutions to (2) is justified by the fact that a lot of mathematical models of physical and technical phenomena involve nonlinear elliptic problems. In this paper we shall present methods based on the variational calculus, which is of significant importance in many disciplines of science and the starting point for various approximate numerical schemes such as Ritz, finite difference, and finite element methods. There are a number of papers studying the elliptic partial differential equations in the divergence form, see, among others, [1], [2], [6], [7], or [8]. In our case, $G$ is not smooth enough to apply [7] ( $G$ should be of class $C(\bar{\Omega} \times R, R))$ or [8], where the existence result follows from the existence of a solution of an associated symmetrized semilinear problem for $G_{x}(y, \cdot)$ continuous on $R$ and satisfying, among others, the estimation

$$
G_{x}(y, x) \leq \alpha\left\{-\theta_{2}(-x) f_{2}(y)\right\} \text { for all } x \leq 0
$$

for nondecreasing $\theta_{2} \in C\left(R_{+}, R_{+}\right)$, nonnegative $f_{2} \in L^{p}\left(\Omega, R_{+}\right), \max \{n / q, 1\}<p \leq \infty$, $\theta_{2}(0)>0$. Here we do not impose either continuity of $G_{x}$ in $x$ on the whole line or conditions similar to (3).

Analogous problems in variational form were treated by means of the sub-, supersolution arguments, for example in [3], [4], [5], [9], [10], [11]. A. Baalal and N. B. Rhouma in [1] also apply variational methods and the comparison principle for sub- and supersolutions, proved in the first part of their paper, to obtain the existence of a weak solution for the second order quasilinear elliptic equation of the form

$$
L(u)(y) \equiv-\operatorname{div}(A(y, u(y), \nabla u(y)))+B(y, u(y), \nabla u(y))=0
$$

for $\Omega \subset R^{n}$ being a bounded domain with smooth boundary and $A(x, u, \nabla u)=\left\{A^{i}(x, u\right.$, $\nabla u)\}_{i=1 . . n}, A^{i}, B: R^{d} \times R \times R^{d} \rightarrow R$. These results do not cover situations described in this paper. This is due to the fact that the authors consider the case when $A^{i}, B$ are Carathéodory functions satisfying the following conditions: for all $\zeta \in R, \xi, \xi^{\prime} \in R^{d}$

$\mathbf{P 1}|A(y, \zeta, \xi)| \leq k_{0}(y)+b_{0}(y)|\zeta|^{p-1}+a|\xi|^{p-1}$,

P2 $\left(A(y, \zeta, \xi)-A\left(y, \zeta, \xi^{\prime}\right)\right)\left(\xi-\xi^{\prime}\right)>0$, if $\xi \neq \xi,^{\prime}$ 
P3 $A(y, \zeta, \xi) \xi \geq \alpha|\xi|^{p}-d_{0}(y)|\zeta|^{p}-e(y)$,

$\mathbf{P 4}|B(y, \zeta, \xi)| \leq k(y)+b(y)|\zeta|^{\alpha}+c|\xi|^{r}$,

with positive constants: $a, c, \alpha, 0<r<\frac{p}{\left(p^{*}\right)^{\prime}}, p^{\prime}=\frac{p}{p-1}, p^{*}=\frac{d p}{d-p}$, and $k_{0} \in L^{p^{\prime}}, b_{0} \in$ $L^{\frac{d}{p-1}}, k \in L^{q}$, where $\left(p^{*}\right)^{\prime}<q<\left(\frac{d}{p-\varepsilon} \wedge \frac{p}{r}\right), d_{0}, e, b \in L^{\frac{d}{p-\varepsilon}}, 0<\varepsilon<1$. Here, the function $B(y, u, \nabla u)=-G_{x}(y, u)$ is not smooth enough. Moreover we omit growth conditions, so (P4) does not necessarily hold in our case. It is worth noting that all properties of $G$ concern the interval $\widetilde{I}$. In the vast existing literature devoted to similar problems, authors usually impose smoothness, convexity or growth conditions on $R$. It turns out that weaker assumptions made on $G$ are still sufficient to deduce the existence of a countable set of positive solutions for (2). Since we shall propose an approach to solvability of our problem based on variational methods, including a dual least action principle, we treat (2) as the Euler-Lagrange equation for the functional

$$
J(x)=\int_{\Omega}\{H(y, \nabla x(y))-G(y, x(y))\} d y
$$

defined on a certain subset $X$ (given later) of $W_{0}^{1, q}(\Omega, R)$. This restriction is associated with the fact that in the general case described by our hypotheses, $J$ is not necessarily bounded on $W_{0}^{1, q}(\Omega, R)$. We shall consider the set $X$ on which $J: X \rightarrow R$ is bounded below. Since our assumptions are not strong enough, we will not use methods like mountain pass lemma (see e.g. [12], [14], [15]), Morse theory and its generalization or the saddle points to investigate critical points of $J$. More precisely, we cannot use the mountain pass theorem, for example, because $G$ is not sufficiently smooth and it does not satisfy any growth conditions; we also omit additional relations similar to the following: there exists $\mu>0$ such that $0<\mu G(y, x) \leq x G_{x}(y, x)$ outside the ball with radius $r \geq 0$. In consequence, $J$ does not satisfy, in general, the (PS)-condition.

This paper is organized as follows: first we introduce the set $X$ of arguments of the action functional $J$ and prove some important properties of $X$. In Section 2 we develop a duality theory, which is based on the idea of using the Fenchel conjugate of functions $G$ and $H$ to define the dual functional. The duality relates the infimum on $X$ of the energy functional associated with our problem, to the infimum of the dual functional on a corresponding set $X^{d}$. The links between minimizers of both functionals give a variational principle and, in consequence, their relation to the boundary value problem. As another consequence of the duality we obtain a numerical version of the variational principle, which gives a measure of the duality gap between the primal and dual functional for approximate solutions to (2). These numerical results are used in the proof of the following existence theorem:

ThEOREM. There exists $x_{0}$ such that $0 \leq x_{0} \leq z_{1}$ a.e. on $\Omega$, and

$$
\left\{\begin{array}{l}
-\operatorname{div}\left(H_{z}\left(y, \nabla x_{0}(y)\right)\right)=G_{x}\left(y, x_{0}(y)\right) \quad \text { for a.e. } y \in \Omega, \\
x_{0} \in W_{0}^{1, q}(\Omega, R) \text { and } \operatorname{div}\left(H_{z}\left(\cdot, \nabla x_{0}(\cdot)\right)\right) \in L^{\infty}(\Omega, R) .
\end{array}\right.
$$

Section 5 is devoted to the existence of a countable set of solutions to (2). Our approach allows us to get information on the least number of solutions by applying the above 
theorem repeatedly for a sequence of disjoint sets $X_{i}$ described by a sequence of intervals, on which $G$ satisfies conditions analogous to (G1)-(G4).

Now we shall construct the set of arguments of $J$ as follows: $X=\left\{x \in W_{0}^{1, q}(\Omega, R), 0 \leq x(y) \leq z_{1}(y)\right.$ a.e. on $\Omega$ and $\left.\operatorname{div}\left(H_{z}(y, \nabla x(y))\right) \in L^{\infty}(\Omega, R)\right\}$. To prove an auxiliary lemma concerning properties of $X$ we use some results due to A. Baalal and N. B. Rhouma, so we cite the relevant definitions and theorem from [1]. DEFINITION 1 . We say that $x$ is a solution of $(6)$ with the boundary value $g \in W^{1-\frac{1}{p}}(\partial \Omega)$, $1<p<\infty$, if

$$
\begin{gathered}
x \in W^{1, p}(\Omega, R), \quad B(\cdot, x, \nabla x) \in L^{(p *)^{\prime}}(\Omega, R), \quad x=g \in W^{1-\frac{1}{p}}(\partial \Omega), \\
\int_{\Omega} A(\cdot, x(y), \nabla x(y)) \nabla \varphi(y) d y+\int_{\Omega} B(y, x(y), \nabla x(y)) \varphi(y) d y=0
\end{gathered}
$$

for all $\varphi \in W_{0}^{1, p}(\Omega, R)$.

DEFINITION 2. We say that $x$ is an upper supersolution of (6) with the boundary value $g \in W^{1-\frac{1}{p}}(\partial \Omega), 1<p<\infty$, if

$$
\begin{gathered}
x \in W^{1, p}(\Omega, R), \quad B(\cdot, x, \nabla x) \in L^{(p *)^{\prime}}(\Omega, R), \quad x \geq g \in W^{1-\frac{1}{p}}(\partial \Omega), \\
\int_{\Omega} A(\cdot, x(y), \nabla x(y)) \nabla \varphi(y) d y+\int_{\Omega} B(y, x(y), \nabla x(y)) \varphi(y) d y \geq 0
\end{gathered}
$$

for all $\varphi \in W_{0}^{1, p}(\Omega, R)$ such that $\varphi \geq 0$.

Similarly, a lower subsolution is characterized by the reverse signs in the above definition.

TheOREM 1. Suppose that (6) admits a bounded lower subsolution $u$ and a bounded upper supersolution $v$ such that $u \leq v$. Then there exists a solution $w$ of (6) such that $u \leq w \leq v$ a.e. on $\Omega$.

Now we shall prove

Lemma 1. $X \neq \emptyset$ and $X$ has the following property: for every $x \in X$, there exists $\widetilde{x} \in X$ such that

$$
-\operatorname{div}\left(H_{z}(y, \nabla \widetilde{x}(y))=G_{x}(y, x(y)) \text { for a.e. } y \in \Omega .\right.
$$

Proof. Let us note that $z_{0} \in X$, which is due to (G1), so $X \neq \emptyset$. Fix $x \in X$. By (G2) and (G1) we have $G_{x}(\cdot, x(\cdot)) \in L^{\infty}(\Omega, R)$. Let us consider (6) with $p=q, g \equiv 0$, $A(y, \zeta, \xi)=H_{z}(y, \xi)$ and $B(y, \zeta, \xi)=-G_{x}(y, x(y))$ :

$$
\left\{\begin{array}{l}
-\operatorname{div}\left(H_{z}(y, \nabla \widetilde{x}(y))=G_{x}(y, x(y)) \quad \text { for a.e. } y \in \Omega\right. \\
x \in W_{0}^{1, p}(\Omega, R) .
\end{array}\right.
$$

Since $\operatorname{div}\left(H_{z}(y, 0)\right)=0$ and $G_{x}(y, x(y)) \leq 0$ a.e. on $\Omega, u=0$ is a bounded lower subsolution of (7). On the other hand, for all nonnegative $\varphi \in W_{0}^{1, q}(\Omega, R)$,

$$
\begin{aligned}
\int_{\Omega} H_{z}\left(y, \nabla z_{0}(y)\right) & \nabla \varphi(y) d y-\int_{\Omega} G_{x}(y, x(y)) \varphi(y) d y \\
& \geq-\int_{\Omega} \operatorname{div}\left[H_{z}\left(y, \nabla z_{0}(y)\right)\right] \varphi(y) d y-\int_{\Omega} G_{x}\left(y, z_{1}(y)\right) \varphi(y) d y \geq 0 .
\end{aligned}
$$


So $z_{0}$ is a bounded upper supersolution of (7). Therefore Theorem 1 yields the existence of a solution $\bar{x}$ to $(7)$ such that $0 \leq \bar{x} \leq z_{0}$ a.e. on $\Omega$ and further $0 \leq \bar{x} \leq z_{1}$ a.e. on $\Omega$. Summarizing: $\bar{x} \in W_{0}^{1, q}(\Omega, R), 0 \leq \bar{x} \leq z_{1}$ a.e. on $\Omega$, and $-\operatorname{div}\left(H_{z}(\cdot, \nabla \bar{x}(\cdot))\right) \in L^{\infty}(\Omega, R)$. Thus $\bar{x} \in X$ and, in consequence, $X$ has the property (D).

2. Duality result. In this section we shall develop the duality, which describes the relations between the critical value of $J$ and the infimum of the dual functional $J_{D}$ : $X^{d} \rightarrow R$ defined as follows:

$$
J_{D}(p)=\int_{\Omega}\left\{-H^{*}(y, p(y))+G^{*}(y,-\operatorname{div} p(y))\right\} d y
$$

with $G^{*}(y, \cdot)$ and $H^{*}(y, \cdot)(y \in \Omega)$ denoting the Fenchel conjugate of $\widetilde{G}(y, \cdot)$ and $H(y, \cdot)$, respectively, where

$$
\widetilde{G}(y, x)= \begin{cases}G(y, x) & \text { if } x \in \tilde{I} \text { and } y \in \Omega \\ \infty & \text { if } x \notin \tilde{I} \text { and } y \in \Omega\end{cases}
$$

and

$$
\begin{aligned}
& X^{d}:=\left\{p \in L^{q^{\prime}}\left(\Omega, R^{n}\right) ; \text { there exists } x \in X\right. \text { such that } \\
&\left.p(y)=H_{z}(y, \nabla x(y)) \text { for a.e. } y \in \Omega\right\} .
\end{aligned}
$$

Lemma 1 leads to the observation that for each $x \in X$ there exists $\widetilde{x} \in X$ satisfying (D). Taking $\bar{p}(\cdot)=H_{z}(\cdot, \nabla \bar{x}(\cdot)) \in L^{q^{\prime}}\left(\Omega, R^{n}\right)$ we infer that $\bar{p} \in X^{d}$. Finally, we can formulate the following

REMARK 1. For every $x \in X$, there exists $p \in X^{d}$ satisfying

$$
-\operatorname{div} p(y)=G_{x}(y, x(y)) \text { for a.e. } y \in \Omega \text {. }
$$

Now we need a kind of perturbation of $J$ and convexity of functions considered on a whole space, so that we use the extension $\widetilde{G}$ of $G$ to the set $\Omega \times R^{n}$ and consider for each $x \in X$ the perturbation $J_{x}: L^{q}(\Omega, R) \rightarrow R$ of the functional $J$ given by

$$
J_{x}(g)=\int_{\Omega}\{-H(y, \nabla x(y))+\widetilde{G}(y, g(y)+x(y))\} d y .
$$

Due to the fact that $\widetilde{G}(y, x(y))=G(y, x(y))$ for a.e. $y \in \Omega$ on $X$, we will not change the notation for the functional $J$ containing $G$ or $\widetilde{G}$.

For every $x \in X$ we define a type of conjugate $J_{x}^{\#}: X^{d} \rightarrow R$ of $J_{x}$ as follows:

$$
J_{x}^{\#}(p)=\sup _{g \in L^{q}(\Omega, R)} \int_{\Omega}\{<g(y), \operatorname{div} p(y)>-\widetilde{G}(y, g(y)+x(y))+H(y, \nabla x(y))\} d y,
$$

which can be simplified to

$$
J_{x}^{\#}(p)=\int_{\Omega}\left\{G^{*}(y, \operatorname{div} p(y))+H(y, \nabla x(y))-<x(y), \operatorname{div} p(y)>\right\} d y .
$$

Lemma 2. For all $p \in X^{d}$

$$
\sup _{x \in X}\left(-J_{x}^{\#}(-p)\right)=-J_{D}(p),
$$

and for each $x \in X$

$$
\sup _{p \in X^{d}}\left(-J_{x}^{\#}(-p)\right)=-J(x) .
$$


Proof. By the definition of $X^{d}$, we infer that for each $p \in X^{d}$ there exists $\bar{x} \in X$ satisfying the equality $p(y)=H_{z}(y, \nabla \bar{x}(y))$ for a.e. $y \in \Omega$ and further, by the assumptions made on $H$

$$
\begin{aligned}
\int_{\Omega} H^{*}(y, p(y)) d y & =\int_{\Omega}\{<\nabla \bar{x}(y), p(y)>-H(y, \nabla \bar{x}(y))\} d y \\
& \leq \sup _{x \in X} \int_{\Omega}\{<\nabla x(y), p(y)>-H(y, \nabla x(y))\} d y \leq \int_{\Omega} H^{*}(y, p(y)) d y .
\end{aligned}
$$

Consequently

$$
\begin{aligned}
\sup _{x \in X}\left(-J_{x}^{\#}(-p)\right) & =\sup _{x \in X} \int_{\Omega}\left\{<\nabla x(y), p(y)>-H(y, \nabla x(y))-G^{*}(y,-\operatorname{div} p(y))\right\} d y \\
& =-J_{D}(p),
\end{aligned}
$$

which is our first assertion. To show the other equality fix $x \in X$ and use Remark 1, which gives the existence of $p$ belonging to $X^{d}$ and such that $-\operatorname{div} p(y)=G_{x}(y, x(y))$ for a.e. $y \in \Omega$. Arguments similar to those in the proof of (10) give

$$
\begin{aligned}
\sup _{p \in X^{d}}\left(-J_{x}^{\#}(-p)\right) & =\sup _{p \in X^{d}} \int_{\Omega}\left\{<x(y),-\operatorname{div} p(y)>-G^{*}(y,-\operatorname{div} p(y))-H(y, \nabla x(y))\right\} d y \\
& =-J(x) .
\end{aligned}
$$

Let us note that, due to the properties of $X$ and $X^{d}$ (Lemma 1 and Remark 1), we were able to avoid calculation of the conjugate with respect to a nonlinear space. As a consequence of both assertions given in the previous lemma we get the duality principle:

THEOREM 2.

$$
\inf _{x \in X} J(x)=\inf _{p \in X^{d}} J_{D}(p)
$$

3. Necessary conditions and regularity. Applying the results of the previous section we shall describe the relation of the minimizers of functionals $J$ and $J_{D}$ to our boundary value problem.

Theorem 3. If $\bar{x} \in X$ satisfies $J(\bar{x})=\inf _{x \in X} J(x)$, then there exists $\bar{p} \in X^{d}$ which is a minimizer of $J_{D}: X^{d} \rightarrow R$ and

$$
\left\{\begin{array}{l}
-\operatorname{div}(\bar{p}(y))=G_{x}(y, \bar{x}(y)) \\
\bar{p}(y)=H_{z}(y, \nabla \bar{x}(y))
\end{array} \quad \text { a.e. on } \Omega .\right.
$$

Proof. By Remark 1 there exists $\bar{p} \in X^{d}$ such that

$$
\int_{\Omega}\left\{<\bar{x}(y),-\operatorname{div} \bar{p}(y)>-G^{*}(y,-\operatorname{div} \bar{p}(y))\right\} d y=\int_{\Omega} G(y, \bar{x}(y)) d y,
$$

which gives

$$
J_{\bar{x}}^{\#}(-\bar{p})+J_{\bar{x}}(0)=0 .
$$

Using the above assertion and the equalities $J_{\bar{x}}(0)=-J(\bar{x})$ and (10) we can derive that

$$
-J(\bar{x})=-J_{\bar{x}}^{\#}(-\bar{p}) \leq \sup _{x \in X}\left(-J_{x}^{\#}(-\bar{p})\right)=-J_{D}(\bar{p}) .
$$


Finally, $J_{D}(\bar{p}) \leq J(\bar{x})$. Now Theorem 2 leads to the conclusion that $\bar{p}$ is a minimizer of $J_{D}: X^{d} \rightarrow R$.

To end the proof it is sufficient to show that $\bar{p}(y)=H_{z}(y, \nabla \bar{x}(y))$ a.e. on $\Omega$. Let us note that $(12)$ and the equalities $J_{\bar{x}}(0)=-J(\bar{x})=-J_{D}(\bar{p})$ yield

$$
J_{\bar{x}}^{\#}(-\bar{p})-J_{D}(\bar{p})=0,
$$

and further

$$
\int_{\Omega}\left\{H^{*}(y, \bar{p}(y))+H(y, \nabla \bar{x}(y))-<\nabla \bar{x}(y), \bar{p}(y)>\right\} d y=0 .
$$

The above assertion, assumption (H1) and the properties of the subdifferential yield

$$
\bar{p}(y)=H_{z}(y, \nabla \bar{x}(y)) \text { a.e. on } \Omega,
$$

which is our claim.

Now we establish a kind of the numerical version of the above variational principle.

TheOREM 4. Assume that $\left\{x_{m}\right\}_{n \in N} \subset X$ is a minimizing sequence of $J: X \rightarrow R$. Then for each $m \in N$ there exists $\left\{p_{m}\right\}_{m \in N} \subset X^{d}$ which is a minimizing sequence of $J_{D}: X^{d} \rightarrow R$ such that

$$
\lim _{m \rightarrow \infty} \int_{\Omega}\left\{H^{*}\left(y, p_{m}(y)\right)+H\left(y, \nabla x_{m}(y)\right)-<p_{m}(y), \nabla x_{m}(y)>\right\} d y=0
$$

and for all $m \in N$

$$
-\operatorname{div} p_{m}(y)=G_{x}\left(y, x_{m}(y)\right) \text { a.e. on } \Omega .
$$

Moreover, for each $\varepsilon>0$, there exists $m_{0} \in N$ such that for all $m>m_{0}$

$$
\left|J_{D}\left(p_{m}\right)-J\left(x_{m}\right)\right| \leq \varepsilon .
$$

Proof. First we establish a lower estimate for $J: X \rightarrow R$. From the definition of $X$ we have that $0 \leq x \leq z_{1}$ for all $x \in X$, so the monotonicity of $G$ implies

$$
\begin{aligned}
J(x) & \geq \int_{\Omega}\left\{\frac{b_{1}}{q}|\nabla x(y)|^{q}+k_{1}(y)-G(y, x(y))\right\} d y \\
& \geq-\int_{\Omega}\left(G\left(y, z_{1}(y)\right)+k_{1}(y)\right) d y>-\infty,
\end{aligned}
$$

and finally $\inf _{m \in N} J\left(x_{m}\right)=: c>-\infty$. Thus for a given $\varepsilon>0$, there exists $m_{0} \in N$ such that for all $m>m_{0}$

$$
c+\varepsilon>J\left(x_{m}\right) \text {. }
$$

By arguments similar to those in the proof of Theorem 3 we state that for each $m \in N$ there exists $p_{m} \in X^{d}$ satisfying (15) and

$$
J_{x_{m}}(0)+J_{x_{m}}^{\#}\left(-p_{m}\right)=0
$$

Our task is now to show that $\left\{p_{m}\right\}_{m \in N}$ is a minimizing sequence for $J_{D}: X^{d} \rightarrow R$. Combining (18), the equalities: $J_{x_{m}}(0)=-J\left(x_{m}\right),(19)$ and (11) we may deduce that for all $m>m_{0}$

$$
c+\varepsilon>J\left(x_{m}\right)=J_{x_{m}}^{\#}\left(-p_{m}\right) \geq \inf _{x \in X}\left(J_{x}^{\#}\left(-p_{m}\right)\right)=J_{D}\left(p_{m}\right)
$$


On the other hand, by Theorem 2 we get $J_{D}\left(p_{m}\right) \geq c$ for all $m \in N$. Summarizing, $\inf _{p \in X^{d}} J_{D}(p)=\inf _{m \in N} J_{D}\left(p_{m}\right)=c$. From the above reasoning and the fact that $J_{x_{m}}^{\#}\left(-p_{m}\right) \leq c+\varepsilon$ for all $m>m_{0}$ we obtain (16), and the auxiliary assertion

$$
J_{x_{m}}^{\#}\left(-p_{m}\right)-J_{D}\left(p_{m}\right) \leq \varepsilon
$$

which gives (14).

4. The existence results. In the sequel we shall prove the existence of a minimizer of $J$ belonging to the set $X$ and being a solution of (2).

THEOREM 5. There exists a minimizer $x_{0}$ of $J$ on $X$ such that $x_{0}$ satisfies (2).

Proof. To prove the existence of the minimizer of $J$ on $X$ we shall employ the scheme presented in [13]. We start our proof with the observation that the set $P_{\widetilde{a}}=\{x \in X ; \widetilde{a} \geq$ $J(x)\}$ is nonempty for sufficiently large $\widetilde{a} \in R$, which follows from the conditions made on $G$ and $H$. Choosing a minimizing sequence $\left\{x_{m}\right\}_{m \in N}$ for $J$ from $P_{\widetilde{a}}$ and applying (17) we infer the boundedness of $\left\{\nabla x_{m}\right\}_{m \in N}$ with respect to the norm $\|\cdot\|_{L^{q}\left(\Omega, R^{n}\right)}$, and hence the boundedness of $\left\{x_{m}\right\}_{m \in N}$ in $W_{0}^{1, q}(\Omega, R)$. Due to this fact, we may deduce that (up to a subsequence) $\left\{x_{m}\right\}_{m \in N}$ tends weakly to a certain $x_{0} \in W_{0}^{1, q}(\Omega, R)$. So that we get : $x_{m} \stackrel{\text { strong }}{\rightarrow}{ }_{m \rightarrow \infty} x_{0}$ in $L^{q}(\Omega, R)$ and further, pointwise convergence of a certain subsequence (still denoted by $\left\{x_{m}\right\}_{m \in N}$ ). So

$$
0 \leq x_{0}(y) \leq z_{1}(y) \leq \underset{y \in \Omega}{\operatorname{essup}} z_{1}(y)
$$

for a.e. $y \in \Omega$, and finally $x_{0} \in L^{\infty}(\Omega, R)$. By the properties of $H(y, \cdot), G(y, \cdot)$ we obtain $\liminf _{m \rightarrow \infty} J\left(x_{n}\right) \geq J\left(x_{0}\right)$, so our task is now to show that $x_{0} \in X$. On account of (20) it is sufficient to prove that $\operatorname{div}\left(H_{z}\left(\cdot, \nabla x_{0}(\cdot)\right)\right) \in L^{\infty}(\Omega, R)$. To this end, let us consider a minimizing sequence $\left\{p_{m}\right\}_{m \in N} \subset X^{d}$, described in Theorem 4 , having properties

$$
-\operatorname{div} p_{m}(y)=G_{x}\left(y, x_{m}(y)\right)
$$

for a.e. $y \in \Omega$ and every $m \in N$, and

$$
\lim _{m \rightarrow \infty} \int_{\Omega}\left\{H^{*}\left(y, p_{m}(y)\right)+H\left(y, \nabla x_{m}(y)\right)-<\operatorname{div} p_{m}(y), x_{m}(y)>\right\} d y=0 .
$$

Using the continuity of $G_{x}$ and (21) we get

$$
\lim _{m \rightarrow \infty}\left(-\operatorname{div} p_{m}(y)\right)=\lim _{m \rightarrow \infty} G_{x}\left(y, x_{m}(y)\right)=G_{x}\left(y, x_{0}(y)\right) .
$$

Therefore, we obtain the boundedness of $\left\{\operatorname{div} p_{m}\right\}_{m \in N}$ in $L^{\infty}(\Omega, R)$ and further in $L^{q^{\prime}}(\Omega, R)$. Hence there exists $z \in L^{q^{\prime}}(\Omega, R)$ such that $\operatorname{div} p_{m} \stackrel{\text { weak }}{\longrightarrow}_{m \rightarrow \infty} z$.

On the other hand, the boundedness of $\left\{\nabla x_{m}\right\}_{m \in N}$ in $L^{q}(\Omega, R)$ and $\left\{\operatorname{div} p_{m}\right\}_{m \in N}$ in $L^{\infty}(\Omega, R)$, growth conditions imposed on $H,(22)$ and the properties of the Fenchel conjugate imply the boundedness of $\left\{p_{m}\right\}_{m \in N}$ in $L^{q^{\prime}}\left(\Omega, R^{n}\right)$. Thus there exists a subsequence still denoted by $\left\{p_{m}\right\}_{m \in N}$ weakly convergent to some $p_{0}$ in $L^{q^{\prime}}\left(\Omega, R^{n}\right)$. From the 
above reasoning we derive

$$
\begin{aligned}
\int_{\Omega}<p_{0}(y), \nabla h(y)>d y & =\lim _{m \rightarrow \infty} \int_{\Omega}<p_{m}(y), \nabla h(y)>d y \\
& =-\lim _{m \rightarrow \infty} \int_{\Omega}<\operatorname{div} p_{m}(y), h(y)>d y=-\int_{\Omega}<z(y), h(y)>d y
\end{aligned}
$$

for any $h \in C_{0}^{\infty}(\Omega, R)$, hence the Euler-Lagrange lemma leads to the equality $\operatorname{div} p_{0}(y)=$ $z(y)$ for a.e. $y \in \Omega$. Finally, $\operatorname{div} p_{m} \stackrel{\text { weak }}{\longrightarrow} m \rightarrow \infty \operatorname{div} p_{0}$. Now taking into account $(21)$ and assumption (G2) we can derive that

$$
\begin{aligned}
0 & =\lim _{m \rightarrow \infty} \int_{\Omega}\left\{G^{*}\left(y,-\operatorname{div} p_{m}(y)\right)+G\left(y, x_{m}(y)\right)+<\operatorname{div} p_{m}(y), x_{m}(y)>\right\} d y \\
& \geq \int_{\Omega}\left\{G^{*}\left(y,-\operatorname{div} p_{0}(y)\right)+G\left(y, x_{0}(y)\right)+<\operatorname{div} p_{0}(y), x_{0}(y)>\right\} d y .
\end{aligned}
$$

Thus, by the properties of the Fenchel transform, we have the equality in (24), and, consequently

$$
-\operatorname{div} p_{0}(y)=G_{x}\left(y, x_{0}(y)\right) \text { for a.e. } y \in \Omega \text {. }
$$

By the assumptions made on $H$ and (22) we infer

$$
\begin{aligned}
0 & =\lim _{m \rightarrow \infty} \int_{\Omega}\left\{H^{*}\left(y, p_{m}(y)\right)+H\left(y, \nabla x_{m}(y)\right)-<p_{m}(y), \nabla x_{m}(y)>\right\} d y \\
& \geq \int_{\Omega}\left\{H^{*}\left(y, p_{0}(y)\right)+H\left(y, \nabla x_{0}(y)\right)-<p_{0}(y), \nabla x_{0}(y)>\right\} d y .
\end{aligned}
$$

On account of the last relation and arguments similar to those in the proof of (25), we get

$$
p_{0}(y)=H_{z}\left(y, \nabla x_{0}(y)\right) \text { for a.e. } y \in \Omega \text {. }
$$

Summarizing, $x_{0} \in W_{0}^{1, q}(\Omega, R), 0 \leq x_{0}(y) \leq z_{1}(y)$ a.e. on $\Omega$ and $\operatorname{div}\left(H_{z}\left(\cdot, \nabla x_{0}(\cdot)\right)\right) \in$ $L^{\infty}(\Omega, R)$, so $x_{0} \in X$. Substituting (26) into (25), one can see that $x_{0}$ satisfies (2).

Now we shall give an example of problem (2) with the right-hand side being not necessarily convex and smooth (with respect to the second variable) on the whole positive axis.

EXAMPLE 1. Let us consider the following problem:

$$
\begin{aligned}
-\operatorname{div}\left[|\nabla x(y)|^{2} \nabla x(y)\right]- & \frac{\partial^{2} x}{\partial y_{1}^{2}}(y)\left(y_{2}+18\right)-\frac{\partial^{2} x}{\partial y_{2}^{2}}(y)\left(y_{1}+18\right) \\
& =\frac{(x(y))^{2}}{(4-x(y))(5+x(y))}+(x(y)+1)^{3}\left(2-y_{1}\right)\left(2-y_{2}\right)
\end{aligned}
$$

for a.e. $y \in \Omega:=\left\{\left(y_{1}, y_{2}\right) \in R^{2} ; 0<y_{1}<2\right.$ and $\left.0<y_{2}<2\right\}$. In this case

$$
H(y, z)=\frac{1}{4}|z|^{4}+\frac{1}{2} z_{1}^{2}\left(y_{2}+18\right)+\frac{1}{2} z_{2}^{2}\left(y_{1}+18\right)
$$

and

$$
G(y, x)=\frac{25}{9} \ln |x+5|-\frac{16}{9} \ln |4-x|-x+\frac{1}{4}(x+1)^{4}\left(2-y_{1}\right)\left(2-y_{2}\right)
$$


for $y \in \Omega$, all $x \in R \backslash\{-5,4\}$ and $z=\left(z_{1}, z_{2}\right) \in R^{2}$. It is clear that $H$ satisfies assumptions (H1)-(H3). Our task is now to find $0<z_{0} \leq z_{1}$ on $\Omega$ such that (G1) holds. Let us consider

$$
z_{0}\left(y_{1}, y_{2}\right)= \begin{cases}y_{2}\left(2-y_{1}\right)\left(2-y_{2}\right) & \text { for }\left(y_{1}, y_{2}\right) \in \Omega \text { and } y_{1}>y_{2} \\ y_{1}\left(2-y_{1}\right)\left(2-y_{2}\right) & \text { for }\left(y_{1}, y_{2}\right) \in \Omega \text { and } y_{1} \leq y_{2}\end{cases}
$$

and $z_{1}\left(y_{1}, y_{2}\right)=1.1 z_{0}\left(y_{1}, y_{2}\right)$. It is easy to check that $z_{0} \in W_{0}^{1,4}(\Omega, R), \operatorname{div}\left(H_{z}\left(\cdot, \nabla z_{0}(\cdot)\right) \in\right.$ $L^{\infty}(\Omega, R)$ and

$$
-G_{x}\left(y, z_{1}(y)\right) \geq \operatorname{div}\left(H_{z}\left(y, \nabla z_{0}(y)\right) \text { a.e. in } \Omega .\right.
$$

Moreover, $G(y, \cdot)$ is convex and continuously differentiable in $[-1,3]$ and $0 \leq z_{1}\left(y_{1}, y_{2}\right)$ $<2$ on $\bar{\Omega}$, so we conclude that $G$ satisfies (G1)-(G4). Finally, Theorem 5 leads to the existence of at least one positive solution $\bar{z} \in W_{0}^{1,4}(\Omega, R)$ with $\operatorname{div}\left(H_{z}(y, \nabla \bar{z}(y)) \in\right.$ $L^{\infty}(\Omega, R)$ for $(27)$.

Of course, we can give an example of a problem with nonlinearity being a smooth function (with respect to the second variable) on $R$.

Example 2. The Dirichlet problem for the equation

$-\operatorname{div}\left[|\nabla x(y)|^{2} \nabla x(y)\right]-\frac{\partial^{2} x}{\partial y_{1}^{2}}(y)\left(y_{2}+18\right)-\frac{\partial^{2} x}{\partial y_{2}^{2}}(y)\left(y_{1}+18\right)=(x(y))^{3}+\left(2-y_{1}\right)\left(2-y_{2}\right)$

for a.e. $y \in \Omega:=\left\{\left(y_{1}, y_{2}\right) \in R^{2} ; 0 \leq y_{1} \leq 2\right.$ and $\left.0 \leq y_{2} \leq 2\right\}$, possess at least one positive solution. Indeed, taking $z_{0}$ and $z_{1}$ as above, analysis similar to that in the previous example gives the existence of at least one positive solution $\bar{z} \in W_{0}^{1,4}(\Omega, R)$ with $\operatorname{div}\left(H_{z}(y, \nabla \bar{z}(y)) \in L^{\infty}(\Omega, R)\right.$ for the last equation.

5. The existence of a countable set of positive solutions. To show the existence of a countable set of solutions to our problem, we assume (H1)-(H2) and the conditions analogous to (G1)-(G4):

G1a: there exist $\left\{a_{i}\right\}_{i \in N},\left\{\bar{a}_{i}\right\}_{i \in N} \in W_{0}^{1, q}(\Omega, R), \operatorname{div}\left(H_{z}\left(\cdot, \nabla \bar{a}_{i}(\cdot)\right)\right) \in L^{\infty}(\Omega, R)$ such that for all $y \in \Omega$ and all $i \in K_{+} \subset N$

$$
\begin{gathered}
0<a_{i}(y)<\bar{a}_{i}(y) \text { a.e. in } \Omega \\
G_{x}\left(y, a_{i}(y)\right) \geq-\operatorname{div}\left(H_{z}\left(y, \nabla \bar{a}_{i}(y)\right)\right) \text { a.e. in } \Omega
\end{gathered}
$$

G1b: there exist $\left\{b_{i}\right\}_{i \in N},\left\{\bar{b}_{i}\right\}_{i \in N} \in W_{0}^{1, q}(\Omega, R) \cap L^{\infty}(\Omega, R), \operatorname{div}\left(H_{z}\left(\cdot, \nabla \bar{b}_{i}(\cdot)\right)\right) \in L^{\infty}(\Omega, R)$ such that for all $y \in \Omega$ and all $i \in K_{+}$:

$$
\begin{gathered}
0<\bar{b}_{i}(y)<b_{i}(y) \text { a.e. in } \Omega \\
G_{x}\left(y, b_{i}(y)\right) \leq-\operatorname{div}\left(H_{z}\left(y, \nabla \bar{b}_{i}(y)\right)\right) \text { a.e. in } \Omega
\end{gathered}
$$

G1c: for each $i \in K_{+}$

$$
a_{i}<b_{i}<a_{i+1} \text { a.e. in } \Omega
$$

G2a: for each $i \in K_{+}: G(y, \cdot)$ is continuously differentiable and convex in a closed neighborhood $\tilde{I}_{i}$ of the interval $I_{i}:=\left[0, \operatorname{ess}_{\sup } \in \Omega\left(b_{i}(y)\right]\right.$ and $G(\cdot, x)$ is measurable on $\Omega$ for all $x \in \tilde{I}_{i}$;

G3a: $G_{x}$ is nonnegative in $\widetilde{I}_{i}$ for each $i \in K_{+}$; 
G4a: for all $i \in K_{+}: \int_{\Omega} G\left(y, a_{i}(y)\right) d y>-\infty, \int_{\Omega} G\left(y, b_{i}(y)\right) d y<+\infty$.

Now for each $i \in K_{+}$we shall construct the set $X_{i}$ as follows

$$
\begin{aligned}
X_{i}=\left\{x \in W_{0}^{1, q}(\Omega, R), a_{i}(y) \leq x(y) \leq b_{i}(y) \text { a.e. on } \Omega .\right. \\
\left.\quad \text { and } \operatorname{div}\left(H_{z}(\cdot, \nabla x(\cdot))\right) \in L^{\infty}(\Omega, R)\right\} .
\end{aligned}
$$

Lemma 3. Assume hypotheses ( $\Omega)$, (K), (H1)-(H3) and (G1a)-(G4a). For each $i \in$ $K_{+}: X_{i} \neq \emptyset$ and $X_{i}$ has the property $(D)$.

Proof. It is clear that $b_{i} \in X_{i}$ for each $i \in K_{+}$. Now we follow the scheme employed in the proof of Lemma 1. Fix $i \in K$ and $x \in X_{i}$. Using (G1a) and (G1b) we infer that $a_{i}$ is a bounded lower subsolution and $\bar{b}_{i}$ is a bounded upper supersolution for (7). So Theorem 1 yields the existence of a solution $\bar{x}$ to (7) such that $a_{i} \leq \bar{x} \leq \bar{b}_{i} \leq b_{i}$, and consequently, $\bar{x} \in X_{i}$.

Applying Theorem 5 to the sequence of nonempty sets $X_{i}$ we obtain

Theorem 6. Suppose that $(\Omega),(\boldsymbol{K}),(\boldsymbol{H 1})-(\boldsymbol{H 3})$ and (G1a)-(G5a) hold. Then for each $i \in K_{+}$there exists a minimizer $x_{i} \in X_{i}$ of $J$ on the set $X_{i}$ which is a solution for (2). Moreover for all $i, j \in K_{+}: x_{i} \neq x_{j}$ for $i \neq j$.

\section{References}

[1] A. Baalal and N. B. Rhouma, Dirichlet problem for quasi-linear elliptic equations, Electronic J. Differential Equations 2002, No. 82, 1-18.

[2] A. Benkirane and A. Elmahi, A strongly nonlinear elliptic equation having natural growth terms and $L^{1}$ data, Nonlinear Anal. Ser. A: Theory Methods 39 (2000), 403-411.

[3] S. Carl and H. Diedrich, The weak upper and lower solution method for quasilinear elliptic equations with generalized subdifferentiable perturbations, Appl. Anal. 56 (1995), 263-278.

[4] E. N. Dancer and G. Sweers, On the existence of a maximal weak solution for a semilinear elliptic equation, Differential Integral Equations 2 (1989), 533-540.

[5] J. Deuel and P. Hess, A criterion for the existence of solutions of nonlinear elliptic boundary value problems, Proc. Roy. Soc. Edinburgh Sect. A 74 (1974/1975), 49-54.

[6] C. Ebmeyer and J. Frehse, Mixed boundary value problems for nonlinear elliptic equations with p-structure in nonsmooth domains, Differential Integral Equations 14 (2001), 801820.

[7] D. Gilbarg and N. S. Trudinger, Elliptic Partial Differential Equations of Second Order, Springer, Berlin, 1983.

[8] N. Grenon, Existence and comparison results for quasilinear elliptic equations with critical growth in the gradient, J. Differential Equations 171 (2001), 1-23.

[9] S. Heikkilä and V. Lakshmikantham, Extension of the method of upper and lower solutions for discontinuous differential equations, Differential Equations Dynam. Systems 1 (1993), 73-85.

[10] T. Kura, The weak supersolution-subsolution method for second order quasilinear elliptic equations, Hiroshima Math. J. 19 (1989), 1-36.

[11] V. K. Le and K. Schmitt, On boundary value problems for degenerate quasilinear elliptic equations and inequalities, J. Differential Equations 144 (1998), 170-218. 
[12] J. Mawhin, Problèmes de Dirichlet variationnels non linéaires, Presses de l'Université de Montréal, 1987.

[13] A. Orpel, On solutions of the Dirichlet problem for a class of partial differential inclusions with superlinear nonlinearities, Numer. Funct. Anal. Optim. 23 (2002), 367-381.

[14] P. H. Rabinowitz, Minimax methods in critical point theory with applications to differential equations, CBMS, AMS, University of Miami, 1986.

[15] M. Willem, Minimax Theorems, Progress in Nonlinear Differential Equations and Their Applications 24, Birkhäuser, Boston, 1996. 